\title{
Systems support for virtualizing traditional courses in science and engineering
}

\author{
Thomas Ottmann, Stephan Trahasch and Tobias Lauer \\ Institute of Computer Science, University of Freiburg, Germany
}

\begin{abstract}
Current Internet technology allows traditional campus-based universities to enter the business of distance education. However, they begin to realise not only that the production of rich-media contents for web-based distance courses is extremely expensive, but that offering distance learning also implies that they have to establish new services completely different from their traditional way of teaching. In this paper we argue that the production of e-lectures by presentation recording and the use of e-lectures as the core of web courses may be a possible way out of this trap. We discuss technical requirements influencing the quality of producing and using e-lectures for distance learning
\end{abstract}

Key words: e-lectures, presentation recording, freehand writing

\section{INTRODUCTION}

Campus-based universities are used to offering their courses in the traditional, time and space bound way. In subjects like science and engineering, on-campus teaching follows a well-structured workflow. Experts in the respective fields present contents in ex-cathedra lectures. These lectures are usually not very interactive although students have the opportunity to ask questions. Lectures are accompanied by (bi)weekly assignments which are completed by the students, marked by the lecturer or teaching assistants, and discussed in small groups.

In order to allow their students more flexibility, campus-based universities have begun to develop web-based courses which are supposed not only to replace, partially or fully, the traditional way of teaching, in particular ex-cathedra lectures, they have also started to use web-based

The original version of this chapter was revised: The copyright line was incorrect. This has been corrected. The Erratum to this chapter is available at DOI: 10.1007/978-0-387-35700-3_33 
technology to 'go virtual,' i.e., to use web courses for self-paced distance learning. Within the last four or five years, large projects were started all over Europe with the ambitious long-term goal to establish 'virtual universities' as new branches of the old brick-and-mortar institutions (SVC, 2003; ULI, 2003; vhb, 2003; VIROR, 2003). However, the campus-based universities are beginning to realise that producing rich-media content for web-based distance education is not only very expensive and time consuming but also requires them to completely change the traditional workflow of teaching and to guarantee a full service for the new clientele. In Germany, at least, students do not have to pay tuition fees for their university studies. Moreover, the scaling effects of large numbers of enrolled students in distance courses have proven to be illusionary. Hence, traditional universities run into a serious cost trap when making their first steps into distance education.

A possible way out of this dilemma is to develop distance courses while maintaining the traditional workflow, by turning ex-cathedra lectures into 'electures,' virtualising assignment submission and grading, and organising tutorial guidance over the web. During the last couple of years we have obtained a lot of experience with this way of offering distance education over the Internet. It can drastically reduce the costs of producing multimedia courses and also offers traditional universities new possibilities for profiling, i.e., of better utilizing their specific qualities in research as an advantage on the global educational market. This experience matches insights gained in other related projects, like Diaper (2000), Jesshope (2000), Taylor (2001). However, to assure the quality of the courses produced in this way, a number of requirements must be fulfilled, which are discussed in this paper.

\section{PRODUCTION OF E-LECTURES}

The first step in virtualising traditional university courses is the production of e-lectures. Traditional lectures in class are turned into multimedia material, which is made accessible over the Internet. E-lectures are then enhanced by animations, simulations, tests, and quizzes and thus turned into web courses for distance education. We first describe how electures can be produced.

Apparently, the simplest form of producing an e-lecture is by recording a standard blackboard-and-chalk lecture. In this scenario lecturers use no technical equipment, no digital whiteboard, no special pen, no pointing device, just blackboard and chalk. They can continue their usual way of lecturing and are not distracted by technology. In particular, mathematicians 
and theoretical computer scientists are keen to teach in this traditional mode, because it allows them to develop complicated lines of thought step by step in real time on the blackboard. We have experimented with this approach and have turned such a live lecture into an e-lecture (Realmedia, 2003)by videotaping it and additionally taking digital high-resolution images from the blackboard whenever the lecturer had filled it with his handwriting. These digital images are necessary since video recordings alone do not lead to well readable material. Furthermore, the blackboard shots can be used to structure the talk naturally, similar to structuring a lecture by the sequence of presented slides. Clearly, producing an e-lecture in this way is quite comfortable for the lecturer. However, the production is technically difficult and time consuming. Skilled technicians are needed to produce acceptable versions of the video recording, to take the digital high-resolution blackboard shots, to record the audio, and to integrate the various data streams into a structured e-lecture. Moreover, the result is not completely satisfying. Readability is far from perfect, navigation is difficult, and content retrieval requires the explicit addition of key words etc.

It is quite common nowadays to support teaching in class by slides or direct computer output. The advantage of using slides is that they are fairly 'low-tech' in the sense that the lecturer does not have to fear any technical problems when presenting the slides. Slides can easily be annotated with handwritten notes during the live lecture. A disadvantage is that everything (except the annotations) remains purely static. (You cannot start an animation or simulation on a slide projector, though some lecturers try this by overlaying series of slides!) Direct computer output avoids unnecessary digital-analogue conversions and supports a direct integration of arbitrary application software into a computer presentation. However, it is usually not easy to annotate a computer presentation with handwritten notes or, simply, to use a pointing or highlighting device. This is because current presentation software, such as PowerPoint, still applies the desktop paradigm for interaction. In a lecture setting it is often much more natural to use an interactive screen or whiteboard with pen input in order to interact with the presentation software. The presentation system should provide an integrated recording facility. All data streams presented or generated during a live lecture should be automatically recorded and synchronised with the other data streams such as audio and video of the lecturer and the audience. At the end of a talk, simply pressing a few buttons should be sufficient in order to turn the whole live talk into an e-lecture and to make it available on the web. In a series of projects we have been successfully pursuing this idea. We have developed an electronic note-taking system, the AOF (Authoring on the Fly) system (Muller, 2000), which has been used to record hundreds of 
hours of lectures, and which has recently been turned into a commercial tool.

Once an e-lecture has been created, it can be made available to students over the Internet or on CD-ROM. In a course offered at a university, electures can replace live lectures. Following the traditional workflow of teaching and learning, it is therefore sufficient to virtualise the second phase of the learning cycle (the dialogue phase) and the submission, marking, and return of exercises. Furthermore, tutorial guidance and communication among students and with the lecturer or a tutor has to be organised over the Internet. The feasibility of this method of producing and running a web course is reported in Datta and Ottmann (2001). In Zupancic and Horz (2002) we report on the acceptance among students of this way of teaching and learning. Even students who have attended the 'real' live lecture make extensive use of e-lectures when recalling the content and preparing for examinations.

In the following section, we consider the whole process of producing and using web courses based on e-lectures from a technological point of view and try to identify the crucial parameters which assure the quality of the resulting courses. In particular, we identify a number of deficiencies when using currently available technology.

\section{QUALITY CRITERIA}

\subsection{The lecturer's point of view}

As with traditional lectures, the most important quality parameter for a course in science and technology is the quality of the content. Traditional brick-and-mortar universities are usually proud of their strengths in highly specialised fields of research. They are convinced that strength in research and teaching go hand in hand, as suggested by the ideal of the unity of research and education introduced by Wilhelm von Humboldt some 200 years ago. In particular, the top universities in the world use this as an important competitive advantage on the global educational market ('we offer education by Nobel Laureates...'). When turning classes taught by renowned researchers into equivalent distance courses the personal characteristics of the lectures should be maintained. Therefore, producing e-lectures as described above is the method of choice. The presentation recording system used must, however, support the preservation of the personal characteristics. It should allow the lecturer to annotate graphically prepared slides or even to write on an empty whiteboard. It might even be desirable to 
annotate graphically not only static objects like slides and images but also running movies and animations (think, e.g., of a medicine teacher explaining a new surgery technique by showing a movie and highlighting the crucial spots). While there is a variety of systems which allow graphical annotation of static objects, like PowerPoint or Adobe Acrobat, systems that allow the annotation of dynamic objects are still subject to current research (Lauer et al, 2001).

When using a pen-based interface for presentation, the characteristics of the handwriting of the lecturer should be maintained when presenting and annotating the content as well as during replay of the e-lecture. Surprisingly, there is a lot of knowledge on handwriting recognition and feature extraction, but very little is known about how to generate personalized, readable, compactly stored handwriting produced by a pen on an interactive large screen or on an interactive display. When recording handwritten annotations on presented slides or on an empty screen, not only the result but also the process of generation should be captured as perfectly as possible. This requires the storage of the time stamps of the generation of the pixel trace in such a way that direct random access and a fine-granular, platform-independent replay is possible. In particular, lecturers teaching mathematics are used to the chalk-and-talk style of teaching when explaining long chains of thought in all detail. It is well known that students greatly appreciate if this live event is captured for later offline use, including all the dynamics of generating the written result on the black- (or white)board. Obviously, smoothing, data reduction, random access capbility, and preservation of personal charasteristics of freehand writing are conflicting goals. Moreover, when using a computer for presentation, annotation, and freehand writing during a live lecture one would expect that the electronic substitute for the traditional blackboard should offer more features than just a blackboard or a slide projector: The computational power should allow the recognition of handwriting and provide an interface for delivering handwritten input to application software, e.g., to symbolic computation systems such as Mathematica. Furthermore, it should allow insertions, corrections, and retrieval of handwritten annotations, and provide a variety of gesture recognition features, like recognising and executing corrections and other mark-ups. Currently, there is no such system available on the market comprising all the desired features.

To ensure the best possible quality of content, a teacher using an integrated system for presentation and recording in class must be able to concentrate on the content as much as possible rather than be distracted by handling the technology. The ideal would be a system that is 'invisible' and as unobtrusive as possible: The classroom should 'recognise' when the teacher enters the lecture theatre, all the material to be used during the 
lecture should be automatically delivered to the computational environment in the lecture hall, the walls should be equipped with interactive whiteboards, the lecturer should be provided with 'e-chalk' and an 'e-sponge.' Everything should have the 'look and feel' of the traditional blackboard-andchalk setting. Recently, a lot of significant improvements in this direction have been made. Rear screen whiteboards, like SmartBoard (2003), or less expensive front screen whiteboards, like the Intelliboard (2003) are available for seminar rooms. The advent of interactive displays with pen input, such as Wacom Cintiq (2003) has indicated the breakthrough for the production of e-lectures from live presentations at several German universities. Figure 1 shows such a tablet built into an integrated presentation and recording lectern.

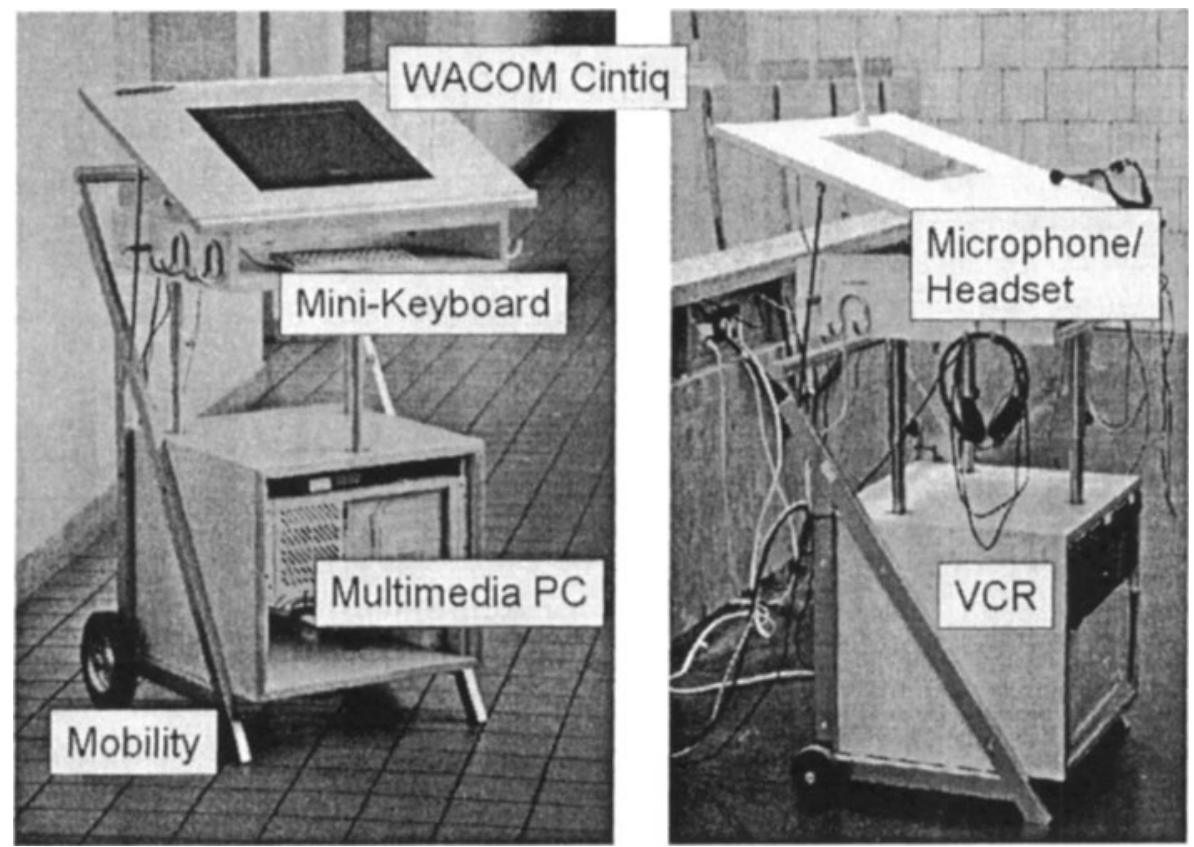

Figure 1. Integrated mobile lectern with built-in Wacom tablet

Once the e-lecture has been recorded, the lecturer may want to add further readings, correct encountered errors, supplement it by quizzes and self-tests, and, finally, integrate it into a complete web course for self-paced study. All these tasks should be conveniently supported by the learning management system. A crucial factor for the acceptance and feasibility of this way of producing an running a web course is that the whole workflow is automated as much as possible. Our experience has shown that a routinely large-scale production of e-lectures by many faculty members rules out manual extra work and time-consuming post-processing. 

engineering

A method to improve the quality is the close contact with the manufacturers of the systems in use. We have formed partnerships with the developers of both the learning management system and the recording software we are using, thus receiving enhanced support and providing them with valuable feedback for future development.

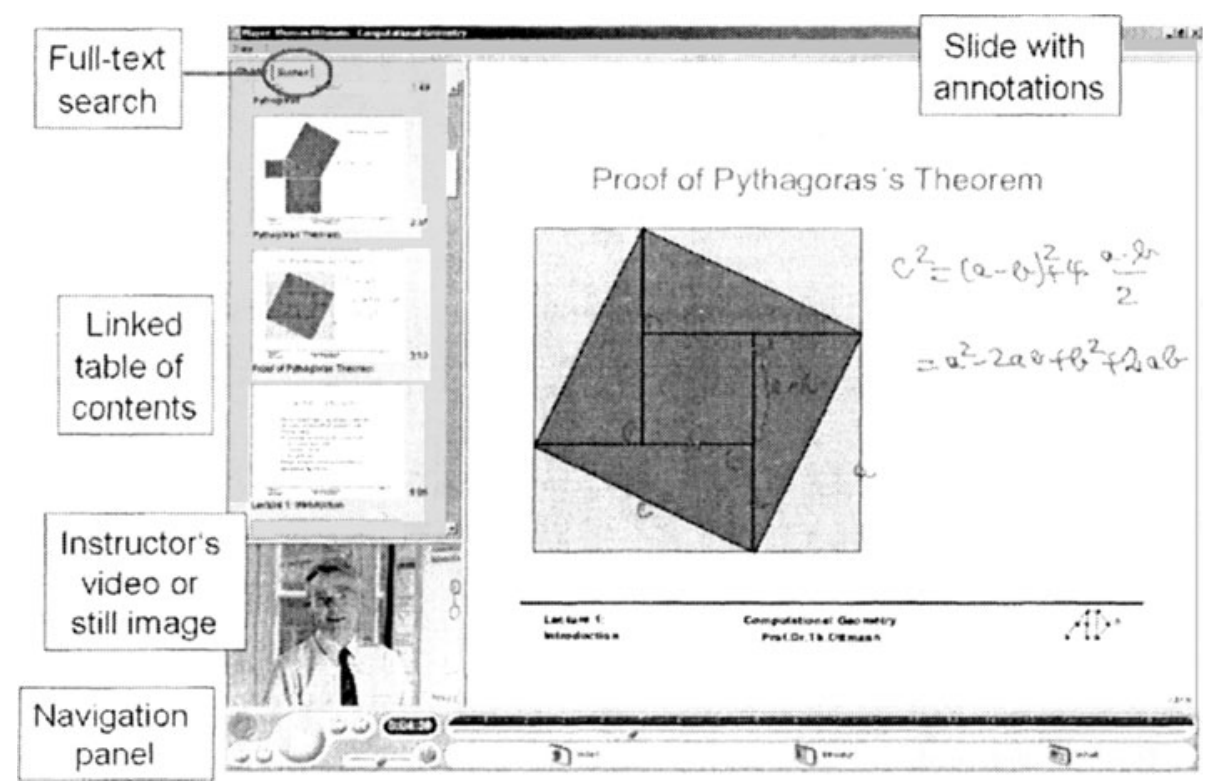

Figure 2. Replay of an e-lecture featuring different ways of navigation.

\subsection{The student's point of view}

From the learner's perspective, the quality of a distance course is not just measured by the contents. On the organizational side, support and tutorial guidance by real people must be available. On the technical side, it must be easy to navigate in an e-learning environment. This is where distance learners playing back an e-lecture could even have an advantage over a students attending the live lecture: they need not stick to the strictly linear nature of the live talk but can pause, skip or repeat passages, directly follow a link to a particular slide from a table of contents, skim the document like a book (by dragging a slider), or search for parts containing certain key words. These navigational features are important quality criteria to assess systems supporting the production of e-lectures. A detailed account can be found in Lauer and Ottmann, (2002), where we have evaluated several existing 
software tools. Note that some features are incompatible, such as 'skimming' a document that is streamed from a server. We have been offering e-lectures in different formats, and it has turned out that most students choose to download the lectures (for better access during replay) rather than have them streamed.

In order to convey as much of the 'live experience' as possible, interstream and intra-stream synchronicity must be maintained during replay independent of the platform running the playback software. If the e-lecture is the only source for getting a 'personal' impression of the lecturer, a video should be included in the e-lecture (see Fig. 2), although experience shows that a 'talking head' does not much contribute to knowledge acquisition.

Unfortunately, no currently available system allows a speeded-up replay, though it is well known that it is possible to follow an audio or video recording if the replay speed exceeds the real time by up to some $30 \%$, and student feedback shows that such a feature would be much appreciated. Furthermore, a quick overview over a large number of e-lectures would be facilitated by (automatically generated) summaries of e-lectures and whole courses. Convenient retrieval and browsing facilities are used for similar tasks. Note that full text search in a repository of e-lectures requires the maintenance of the symbolic representation of the recorded data. Unfortunately, most currently available systems for presentation recording are just frame grabbers, i.e. they take a series of screenshots during the live presentation and turn them into a digital video, thereby destroying the symbolic representation of the recorded data.

\subsection{Organisational and administrative requirements}

An e-lecture can be distributed to students on CD-ROM or it can be used to create an online web course with additional learning resources like selfassessments, animations etc., and communication services like a forum or chat. Therefore a learning management system (LMS) has to be used which makes the material accessible over the Internet and creates a personalised view of the course. To ensure a high failsafe performance, the LMS must be installed and maintained by the university computing centre that can guarantee permanent availability of the system. This is particularly important for the electronic submission of assignments (Kandzia and Trahasch, 2002). It is easier for the students if they only have to use one campus-wide login for all systems including the LMS, which should be integrated in the university-wide IT infrastructure. Access from the LMS to the human resources system of the university is also necessary to avoid redundant information storage. 
E-lectures can also be distributed to and reused by others. This requires indexing with metadata and storage in a digital repository, such as Merlot (2003) or the ARIADNE Knowledge Pool System Ariadne (2003). It is crucial to use a standardised metadata scheme like the LOM (2003) standard for the repository. After an e-lecture has been recorded it has to be indexed by the author. The metadata should be completed and checked by librarians. Only if the metadata satisfies certain quality standards, can the learning resource be stored in the repository and replicated with other repositories so it can be reused for other courses. It remains an open question how quality standards concerning the contents can be ensured.

\section{CONCLUSION}

We have demonstrated that e-lectures produced by recording live presentations and used as core components of web courses can be an efficient way for traditional universities to virtualise their curricula. We have identified crucial factors and deficiencies in current technologies, including an interaction paradigm that does not require instructors to change their teaching habits while recording their lectures, comfortable navigation withing e-lectures for students, and a suitable LMS and content repository supporting standardized metadata schemes to organise and reuse e-lectures.

\section{REFERENCES}

ARIADNE (2003) website. http://www.ariadne-eu.org

Datta, A. and Ottmann, T (2001) Towards a Virtual University. Journal of Universal Computer Science, 7 (10), 2001, 870-885

Diaper, D (2000) One person and Their Dog Performing Electronic Lecturing, in: Jin, Q., Li, J., Zhang, N., Cheng, J., Yu, C. and Noguchi, S. (Eds.) International Conference on Information Society and the 21 st Century (IS2000, Japan), 596-602

Intelliboard (2003) description page. http://www.mcr-gmbh.com/

Jesshope, C. R. (2000) The use of multi-media in internal and extramural teaching, in:

Proceedings of Lifelong Learning Conference, Queensland, Australia, 2000, 257-262

Kandzia, P.-T., Trahasch, S (2002).Tutored Assignments Going Online, Proceedings of the 4th ICNEE, Lugano, Switzerland, May 2002

Lauer, T., Müller, R. and Ottmann, T.(2001) Animations for Teaching Purposes: Now and Tomorrow, Journal of Universal Computer Science, 7, (5), 2001, 420-433

Lauer, T. and Ottmann, T.(2002) Means and Methods in Automatic Courseware Production:

Experience and Technical Challenges, in: Proc. E-Learn 2002, Montréal, Canada

LOM (2003) specification. http://ltsc.ieee.org/wg12/index.html

Merlot (2003) website. http://www.merlot.org 
Müller, R. and Ottmann, T.(2000) The 'Authoring on the Fly' System for Automated Recording and Replay of (Tele)presentations, ACM/Springer Multimedia Systems Journal 8 (3), May 2000

SVC - Swiss Virtual Campus (2003) http://www.virtualcampus.ch

Taylor, J. and Diaper, D.(2001) Maintaining Choice: Electronic University Education and

Diversity, in: Proceedings of the First International Conference on Learning and Teaching

On-line: Practices, Challenges and Prospects. China, January, 2001

RealMedia (2003) e-lecture.

http://www.viror.de/lernen/lernmodule/verschiedenes/ressourcen/

einzelthemen/ottmann_goedel.htm

ULI (2003)(Collaborative University Education in Computer Science). http://uli-campus.de/ vhb (2003) Virtual University Bavaria, http://www.vhb.org

VIROR (2003) Virtual University in the Upper Rhine Valley, website. http:// www.viror.de/ Zupancic, B. and Horz, H.(2002) Lecture Recording and Its Use in a Traditional University

Course. Proceedings of ITiCSE 2002, Aarhus, Denmark, June 2002, ACM Press, 24-28 SmartBoard (2003) SMART Technologies Inc. http://www.smarttech.com Wacomboard (2003) Wacom Technology Co. http://www.wacom.com 